\title{
Der Bildhauer „Moschos Kallippos” aus Synnada Ein kleines Corrigendum zum Künstlerlexikon der Antike
}

\author{
N. Eda AKYÜREK ŞAHİN*
}

Im zweiten Band des im Jahre 2004 erschienen Lexikons „Künstlerlexikon der Antike” schreibt Tomas Lochman ${ }^{1}$ im Lemma „Kallippos” Folgendes²:

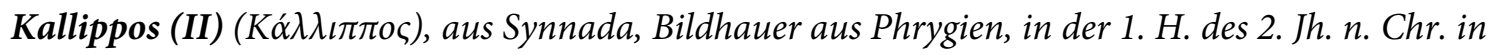
den Marmor-Wkstn bei Dokimeion tätig. Nur bek. durch die Doppel-Sign. auf einer Hera-Statue aus Aspendos im Mus. von Antalya (Inv. Nr. A 97); E. Atalay, Weibliche Gewandstatuen des 2. Jahrhunderts n. Chr. aus ephesischen Werkstätten (Denkschriften Wien 206), W. 1989, 69 s., Nr. 6 Abb. 12), die K. zus. mit seinem Kollegen $\rightarrow$ Moschos gearbeitet hat.

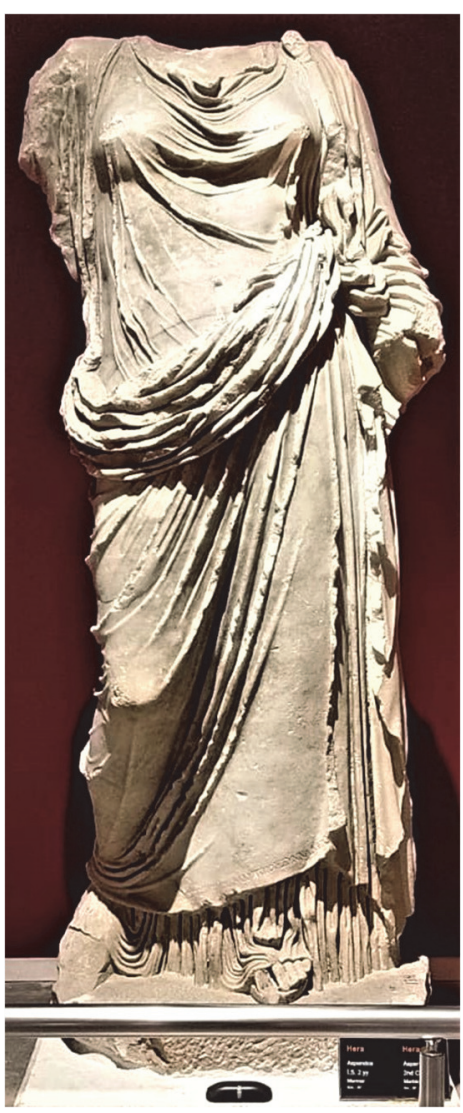

Abb. 1) Hera-Statue

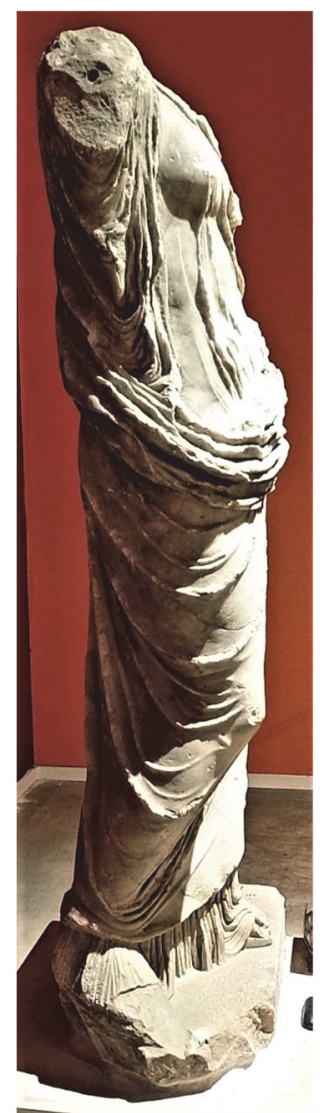

Abb. 2

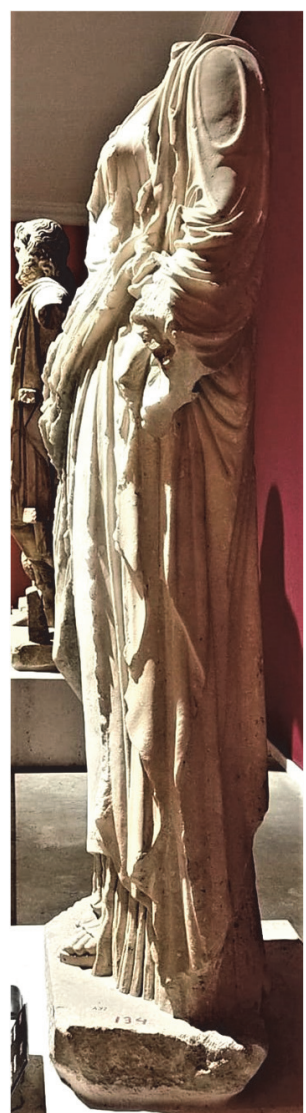

Abb. 3

${ }^{*}$ Prof. Dr. N. Eda Akyürek Şahin, Universität Akdeniz, Fakultät für Human- und Sozialwissenschaften, Fach für Antike Sprachen und Kulturen, TR-07058 Kampus, Antalya (edasahin@akdeniz.edu.tr; https://orcid.org/0000-0002-5993-0566).

Die Fotos stammen aus dem Archiv von Antalya Museum. Ich bedanke mich herzlich bei Herrn Süleyman Atalay, Archäologe im Museum von Antalya, für die Zusendung sämtlicher Fotos.

${ }^{1}$ Tomas Lochman schrieb auch andere Lemmata in diesem Lexikon, s. dazu Lochman 2003, 111, Anm. 18. Eine Zusammenstellung darüber, welche Lemmata ein Autor im Lexikon geschrieben hat, wäre nützlich.

${ }^{2}$ Lochman 2004b, 560. 
Laut Lochman soll ein gewisser „Moschos” aus Phrygien als Kollege mit dem Bildhauer Kallippos aus Synnada in Dokimeion zusammengearbeitet haben. Lochman schreibt im Lemma „Moschos” desselben Lexikons das Folgende 3 :

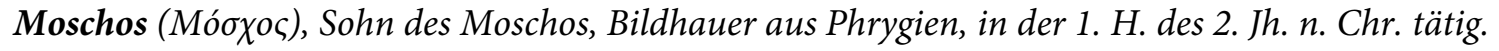
Nur bek. durch die Doppel-Sign. auf einer Hera-Statue aus Aspendos im Mus. von Antalya (E. Atalay, Weibliche Gewandstatuen des 2. Jahrhunderts n. Chr. aus ephesischen Werkstätten [DenkschriftenWien 206], W. 1989, 69 s., Nr. 6 Abb. 12), die M. zus. mit seinem aus Synnada stammenden Kollegen $\rightarrow$ Kallippos (II) (KLA II Addendum) gearbeitet hat. Die beiden Bildhauer sind zweifellos aus den Wkstn in der Nähe der kaiserl. Marmorbrüche bei Dokimeion hervorgegangen, deren administratives Zentrum im benachbarten Synnada, dem Herkunfstort des $\rightarrow$ Kallippos (II) (KLA II, Addendum), lag.

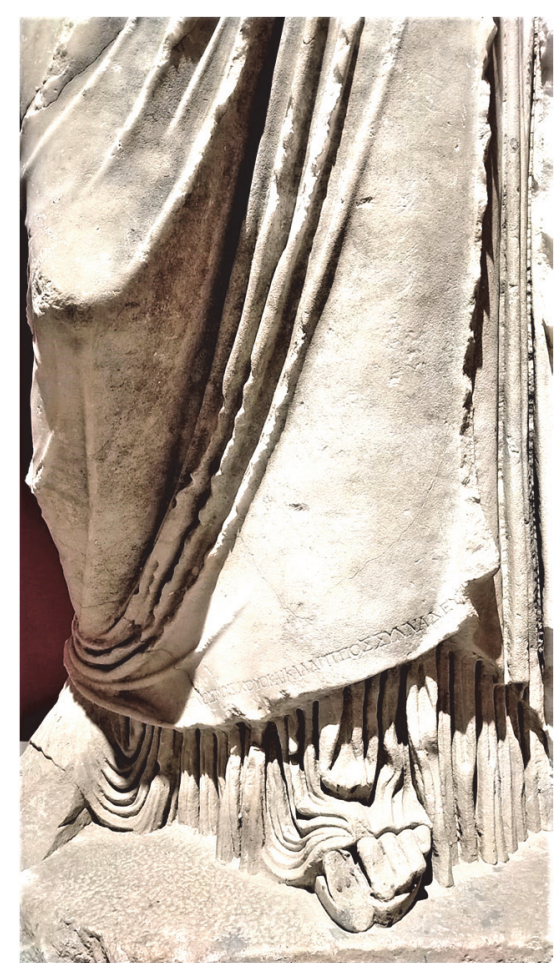

Abb. 4

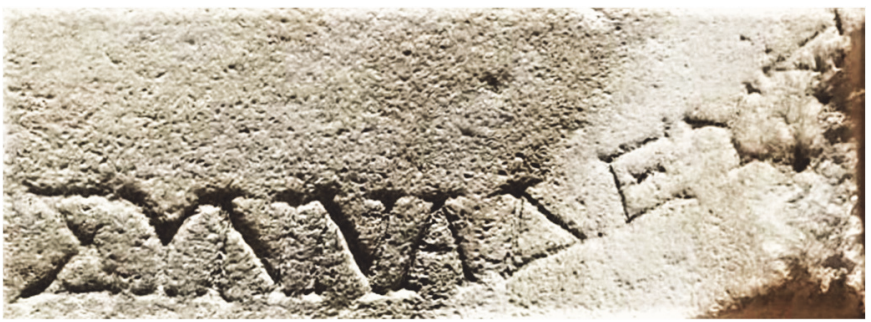

Abb. 5

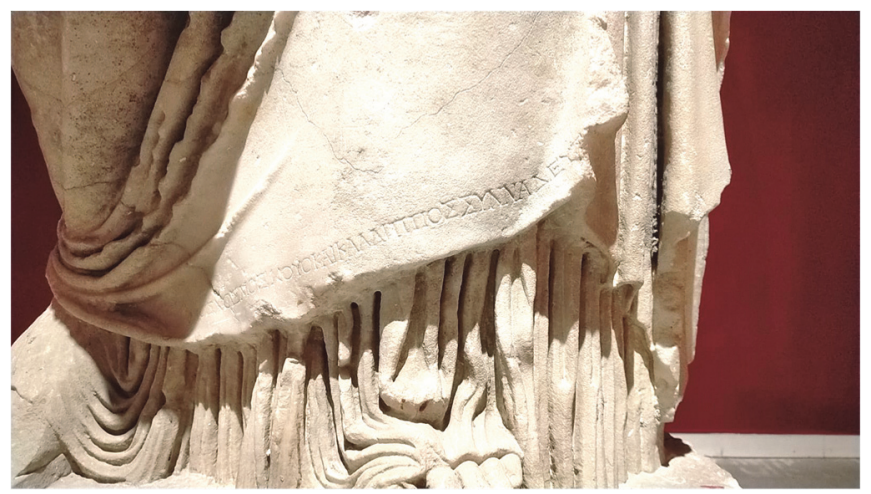

Abb. 6

Diese marmorne Hera-Statue (H: $184 \mathrm{~cm}$ ) aus der ersten Hälfte des 2. Jh. n. Chr. (Abb. 1-5) ist wohl im Jahre $1884^{4}$ in Aspendos gefunden worden und befindet sich jetzt, wie oben angegeben,

${ }^{3}$ Lochman 2004a, 94.

${ }^{4}$ Nimet Arsan schrieb 1946, dass diese Statue zusammen mit einer weiteren weiblichen Gewandstatue und einer Panzerstatue von F. v. Luschan im Jahre 1884 in Aspendos hinter der großen Exedra gefunden wurde, s. Arsan 1946, 443. Sie erhielt diese Information aus dem Buch von Lanckoronski: 1890, 95 mit Abb. 71 und 72 (diese beiden waren in Stücke gebrochene unvollständige Statuen). Lanckoronski erwähnt dort allerdings nichts von der hier behandelten Hera-Statue. Arsan schrieb des Weiteren, dass die gebrochenen Statuen am Fundort gelassen und nur diese Herastatue ins Museum von Antalya gebracht wurde. Anscheinend befand sich die Statue im Jahre 1904 aber noch am Fundort, da R. Norton ihre Inschrift damals dort kopieren konnte und diese dann von van Buren im Jahre 1908 publiziert wurde, 189. Im Museumskatalog von Antalya heißt es, dass diese Statue erst im Jahre 1926 ins Museum kam, s. Demirer - Çınar - Sezgin Karakaş - Koç 2005, 240 Nr. 97. 
im Museum von Antalya (Inv. Nr. A 97). ${ }^{5}$ Sie ist in mehreren Studien behandelt bzw. abgebildet worden. ${ }^{6}$ Auch ihre Inschrift (Abb. 6-9) ist schon publiziert. ${ }^{7}$ Die Signatur des Künstlers befindet sich schräg auf dem unteren Rand des Himations und lautet wie folgt:

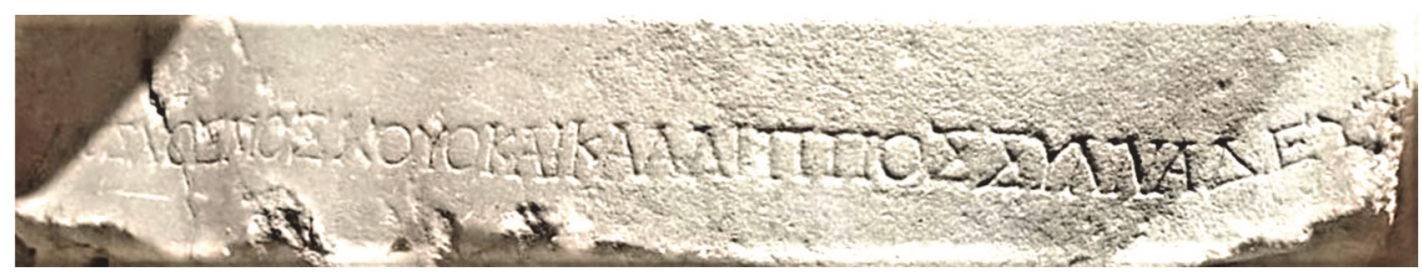

Abb. 7

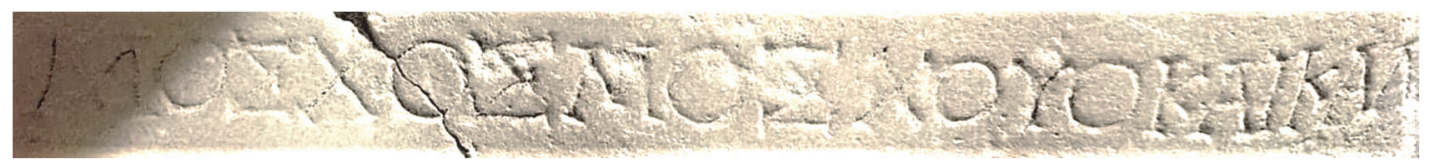

Abb. 8

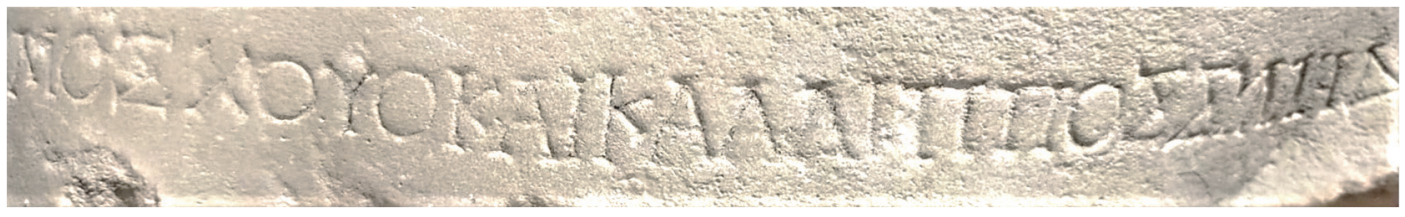

Abb. 9

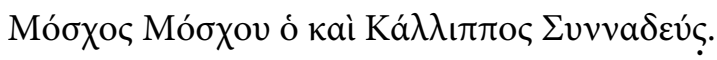

Moschos, der Sohn des Moschos, der auch Kallippos hieß aus Synnada.

Die Inschrift ist hier ganz deutlich: Es handelt sich um einen Künstler aus Synnada, der zwei Namen hatte: Moschos Kallippos. Daher ist das Künstlerlexikon mit der dort geäußerten Trennung der Personen Moschos und Kallippos zu korrigieren. Die epigraphische Publikation der Inschrift war sehr alt $^{8}$; sie wurde erst im Jahre 2020 in einer Studie erneut publiziert, nunmehr mit Foto. ${ }^{9}$ T. Lochman kannte die alte Publikation der Inschrift offensichtlich nicht. D.h. er hatte die Inschrift wohl nicht gesehen, wodurch meiner Meinung nach dieser Fehler entstanden ist.

${ }^{5}$ Das Foto der Statue (nicht aber der Inschrift) ist in den Museumskatalogen abgebildet worden, vgl. z.B. Özgür 1988, 14 Abb. 6; Demirer - Çınar - Sezgin Karakaş - Koç 2005, 101, Nr. 97 und 240 Nr. 97.

${ }^{6}$ Z.B. Arsan 1946, 443-444, Taf. 55, Abb. 10; Kruse 1975, 135, 346-347 Nr. D. 38; Atalay 1989, 69 Abb. 12; Filges 1999, 422 Anm. 206.

${ }^{7}$ Van Buren 1908, 189 (er schreibt das Ethnikon mit einem Ny von der Handkopie von R. Norton, der die Statue wohl noch am Fundort sah [Aspendus, behind the basilica]); Reinach 1909, 316; Demirer - Bru 2020, 276 Anm. 23 und Abb. 3-4 auf Seiten 283-284; Kruse 1975, 346-347 Nr. D. 38. Van Buren verglich diese Inschrift mit CIG, 6970 und IGR III, 1360 (= IG II ${ }^{2}$, 10385). Diese letzte Inschrift ist ein Epigramm aus

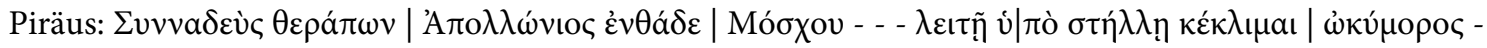

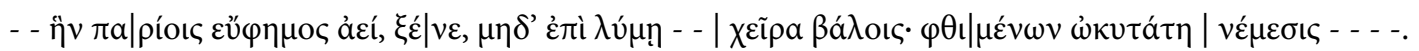

${ }^{8}$ Van Buren 1908, 189 (= Reinach 1909, 316).

${ }^{9}$ Demirer - Bru 2020, 276 Anm. 23 Abb. 4. 


\section{Bibliographie}

Arsan 1946

Atalay 1989

Demirer - Çınar - Sez-

gin Karakaş - Koç 2005

Demirer - Bru 2020

Filges 1999

Kruse 1975

Lanckoronski 1890

Lochman 2003

Lochman 2004a

Lochman 2004b

Özgür 1988

Reinach 1909

Van Buren 1908

Vollkommer 2004
N. Arsan, Anadolu'nun M. S. I. ve II. Yüzyıllarına Ait Giyimli ve Ayakta Duran Kadın Heykelleri, Belleten 10/39, 1946, 425-470, Lev. XLVI-LXVII.

E. Atalay, Weibliche Gewandstatuen des 2. Jahrhunderts n. Chr. aus ephesischen Werkstätten (DenkschriftenWien 206), Wien 1989.

Ü. Demirer - Ü. Çınar - N. Sezgin Karakaş - A. Koç, Antalya Museum, Ankara 2005.

Ü. Demirer - H. Bru, The Statues Dedicated by Pelopidianos in Perge and the Diffusion of the Dokimeion Marble (Perge'de Pelopidianos Tarafindan Adanan Heykeller ve Dokimeion Mermerinin Dağllımı), in: A. Mörel - G. Kaşka - H. Köker - M. Kaşka - M. Fırat - S. O. Akgönül (Hgg.), Pisidia ve Yakın Çevresinde Üretim, Ticaret ve Ekonomi. Uluslararası Sempozyum Bildirileri. Pisidia Araştırmalar1 II. 31 Ekim-03 Kasim 2018, Isparta 2020, 274-284.

A. Filges, Marmorstatuetten aus Kleinasien. Zu Ikonographie, Funktion und Produktion antoninischer, severischer und späterer Idealplastik, IstMitt 44, 1999, 377-430.

H.-J. Kruse, Römische weibliche Gewandstatuen des zweiten Jahrhunderts n. Chr, Göttingen 1975.

K. Lanckoronski, Städte Pamphyliens und Pisidiens. Band I: Pamphylien, Prag-Wien-Leipzig 1890.

T. Lochman, Studien zu kaiserzeitlichen Grab- und Votivreliefs aus Phrygien, Basel 2003.

T. Lochman, in: Vollkommer 2004, 94, s.v. Moschos.

T. Lochman, in: Vollkommer 2004, 560, s.v. Kallippos.

M. E. Özgür, Aspendos. Ein Reiseführer, 1988.

A.-J. Reinach, Bulletin épigraphique (suite et fin), in: Revue des Études Grecques 22/fascicule 98-99, 1909, 306- 335.

A. W. van Buren, Inscriptions from Asia Minor, Cyprus and Cyranaica, JHS 28, 1908, 180-201.

R. Vollkommer (Hrsg.), Künstlerlexikon der Antike 2. L-Z. Addendum A-K, München-Leipzig 2004. 


\section{Synnadalı Heykeltraş "Moskhos Kallippos" \\ Künstlerlexikon der Antike İçin Küçük Bir Düzeltme \\ Özet}

Bu küçük yazıda "Künstlerlexikon der Antike" sözlüğündeki "Kallippos" ve "Moskhos" maddelerinde yapılmış bir hata düzeltilmektedir. Bu maddelerin yazarı T. Lochman Kallippos ve Moskhos'un iki ayrı heykeltraş olduğunu belirtmiş ve her iki isim için ayrı ayrı sözlük maddesi yazmıştır. Lochman'ın temel aldığı yazıt Aspendos'ta bulunmuş ve bugün Antalya Müzesi'nde duran bir Hera heykelinin mantosunun etek kısmına eğri olarak yazılmıştır (bkz. fig. 1-9). Yazıt tamdır ve Moskhos'un oğlu, bir adı da Kallippos olan Synnadalı Moskhos (yaptı) şeklinde Türkçe'ye çevrilebilir. Yani bu Hera heykelini yapan kişi tektir, adı da Moskhos Kallippos'tur. Lochman'ın, olasılıkla yazıtın yayımlı olduğu çok eski yayınları görmediği için hata yaptığı ve yazıtta iki ayrı heykeltraştan bahsedildiğini düşünerek sözlükte de buna göre iki ayrı açıklama verdiği anlaşılmakta ve bu yanılgı düzeltilmektedir.

Anahtar Sözcükler: Künstlerlexikon der Antike, Hera heykeli, Aspendos, Antalya Müzesi, Synnada, heykeltraş.

\section{The sculptor "Moschos Kallippos" from Synnada A small Corrigendum to the Künstlerlexikon der Antike}

Abstract

In this small article, a mistake made in the "Kallippos" and "Moschos" entries in the dictionary "Künstlerlexikon der Antike" is corrected. The author of these entries T. Lochman stated that Kallippos and Moschos were two separate sculptors and consequently wrote a separate dictionary entry for each of them. The inscription on which Lochman based was found in Aspendos and inscribed in a curved manner on the skirt of the mantle of a Hera statue preserved today in the Antalya Museum (see fig. 1-9). The inscription is complete and it reads: Moschos, son of Moschos, also called Kallippos, from Synnada. In other words, the person who made this Hera statue is a single person whose name is Moschos alias Kallippos. It is understood that Lochman made this mistake because he possibly did not see the older publications where the inscription was published, which made him think that two different sculptors were in question. Therefore, he provided two separate entries in the dictionary, a mistake which is corrected now in this contribution.

Keywords: Künstlerlexikon der Antike, Hera statue, Aspendos, Antalya Museum, Synnada, sculptor. 